\title{
Flexible Composition in LTAG: Quantifier Scope and Inverse Linking
}

joint work with Aravind K. Joshi and Maribel Romero

\author{
Laura Kallmeyer \\ Lattice, Paris 7 \\ laura.kallmeyer@linguist.jussieu.fr
}

Réunion Genl, 29.09.2003 


\section{Overview}

- The data: Nested Quantifiers

- The framework:

- LTAG semantics

- Quantifier scope

¿ The solution:

- Flexible composition

- Quantifier set approach

- Conclusion 


\section{Nested Quantifiers (1)}

$\left[\mathrm{Qu}_{1}\left[\mathrm{Qu}_{2}\right]\right]$ : both scope orderings are possible: $\mathrm{Qu}_{1}>\mathrm{Qu}_{2}$ (surface reading) and $\mathrm{Qu}_{2}>\mathrm{Qu}_{1}$ (inverse linking reading).

(1) Every president of an African country came to the meeting.

$\mathrm{Qu}_{1}>\mathrm{Qu}_{2}: \forall x[\exists y[y$ Afr._country $\wedge x$ president_of $y] \rightarrow x$ came to the meeting]

(2) A representative from every African country came to the meeting.

$\mathrm{Qu}_{2}>\mathrm{Qu}_{1}: \forall x[x$ Afr. country $\rightarrow \exists y[y$ repres. from $x] \wedge y$ came to the meeting] 


\section{Nested Quantifiers (2)}

$\mathrm{Qu}_{1} \ldots\left[\mathrm{Qu}_{2}\left[\mathrm{Qu}_{3}\right]\right]$ : the scope readings where $\mathrm{Qu}_{1}$ intervenes between $\mathrm{Qu}_{2}$ and $\mathrm{Qu}_{3}$ are impossible (Hobbs \& Shieber 1987; Larson 1987): 


\section{Nested Quantifiers (2)}

$\mathrm{Qu}_{1} \ldots\left[\mathrm{Qu}_{2}\left[\mathrm{Qu}_{3}\right]\right]$ : the scope readings where $\mathrm{Qu}_{1}$ intervenes between $\mathrm{Qu}_{2}$ and $\mathrm{Qu}_{3}$ are impossible (Hobbs \& Shieber 1987; Larson 1987):

- Possible scope orders:

- $\mathrm{Qu}_{1}>\mathrm{Qu}_{2}>\mathrm{Qu}_{3}$

- $\mathrm{Qu}_{1}>\mathrm{Qu}_{3}>\mathrm{Qu}_{2}$

- $\mathrm{Qu}_{2}>\mathrm{Qu}_{3}>\mathrm{Qu}_{1}$

- $\mathrm{Qu}_{3}>\mathrm{Qu}_{2}>\mathrm{Qu}_{1}$ 


\section{Nested Quantifiers (2)}

$\mathrm{Qu}_{1} \ldots\left[\mathrm{Qu}_{2}\left[\mathrm{Qu}_{3}\right]\right]$ : the scope readings where $\mathrm{Qu}_{1}$ intervenes between $\mathrm{Qu}_{2}$ and $\mathrm{Qu}_{3}$ are impossible (Hobbs \& Shieber 1987; Larson 1987):

- Possible scope orders:

- $\mathrm{Qu}_{1}>\mathrm{Qu}_{2}>\mathrm{Qu}_{3}$

- $\mathrm{Qu}_{1}>\mathrm{Qu}_{3}>\mathrm{Qu}_{2}$

- $\mathrm{Qu}_{2}>\mathrm{Qu}_{3}>\mathrm{Qu}_{1}$

- $\mathrm{Qu}_{3}>\mathrm{Qu}_{2}>\mathrm{Qu}_{1}$

- Impossible scope orders:

- * $\mathrm{Qu}_{2}>\mathrm{Qu}_{1}>\mathrm{Qu}_{3}$

- * $\mathrm{Qu}_{3}>\mathrm{Qu}_{1}>\mathrm{Qu}_{2}$ 


\section{Nested Quantifiers (3)}

(3) Two politicians spy on someone from every city. (Larson 1987) 


\section{Nested Quantifiers (3)}

(4) Two politicians spy on someone from every city. (Larson 1987)

ص * $\mathrm{Qu}_{2} \mathrm{Qu}_{1} \mathrm{Qu}_{3}={ }^{*} \exists 2 \forall$ :

$\exists z\left[\operatorname{person}^{\prime}(z) \wedge 2 x[\right.$ politicians $(x) \wedge$

$\left.\left.\left.\forall y\left[\operatorname{city}^{\prime}(y) \rightarrow \operatorname{from}^{\prime}(z, y)\right] \wedge \operatorname{spy}^{\prime}(x, z)\right]\right]\right]$

Problem: $\operatorname{spy}^{\prime}(x, z)$ in nuclear scope of $\exists z \Rightarrow 2 x$ also in nuclear scope of $\exists z \Rightarrow \forall y$ also in nuclear scope of $\exists z \Rightarrow$ $\operatorname{from}^{\prime}(z, y)$ also in nuclear scope of $\exists z$ Reading can therefore be excluded for logical reasons 


\section{Nested Quantifiers (3)}

(5) Two politicians spy on someone from every city. (Larson 1987)

2 * $\mathrm{Qu}_{2} \mathrm{Qu}_{1} \mathrm{Qu}_{3}={ }^{*} \exists 2 \forall$ :

$\exists z\left[\operatorname{person}^{\prime}(z) \wedge 2 x\left[\right.\right.$ politicians $^{\prime}(x) \wedge$

$\left.\left.\left.\forall y\left[\operatorname{city}^{\prime}(y) \rightarrow \operatorname{from}^{\prime}(z, y)\right] \wedge \operatorname{spy}^{\prime}(x, z)\right]\right]\right]$

Problem: $\operatorname{spy}^{\prime}(x, z)$ in nuclear scope of $\exists z \Rightarrow 2 x$ also in nuclear scope of $\exists z \Rightarrow \forall y$ also in nuclear scope of $\exists z \Rightarrow$ $\operatorname{from}^{\prime}(z, y)$ also in nuclear scope of $\exists z$ Reading can therefore be excluded for logical reasons

ص * $\mathrm{Qu}_{3} \mathrm{Qu}_{1} \mathrm{Qu}_{2}={ }^{*} \forall 2 \exists$ : Inverse linking

$\forall y\left[\operatorname{city}^{\prime}(y) \rightarrow 2 x\left[\right.\right.$ politicians $^{\prime}(x) \wedge$

$\left.\left.\exists z\left[\left[\operatorname{person}^{\prime}(z) \wedge \operatorname{from}^{\prime}(z, y)\right] \wedge \operatorname{spy}^{\prime}(x, z)\right]\right]\right]$ 


\section{LTAG semantics (1)}

Kallmeyer \& Joshi (2003)

- elementary trees are linked to flat semantic representations

a the derivation tree shows how the semantic representations are combined

- Underspecified representations:

- enrich formulas with labels $l_{1}, l_{2}, \ldots$ and holes

$h_{1}, h_{2}, \ldots$ (metavariables ranging over labels)

- scope constraints $x \geq y$ with $x$ and $y$ being labels or holes or variables 


\section{LTAG semantics (2)}

(6) John always laughs.

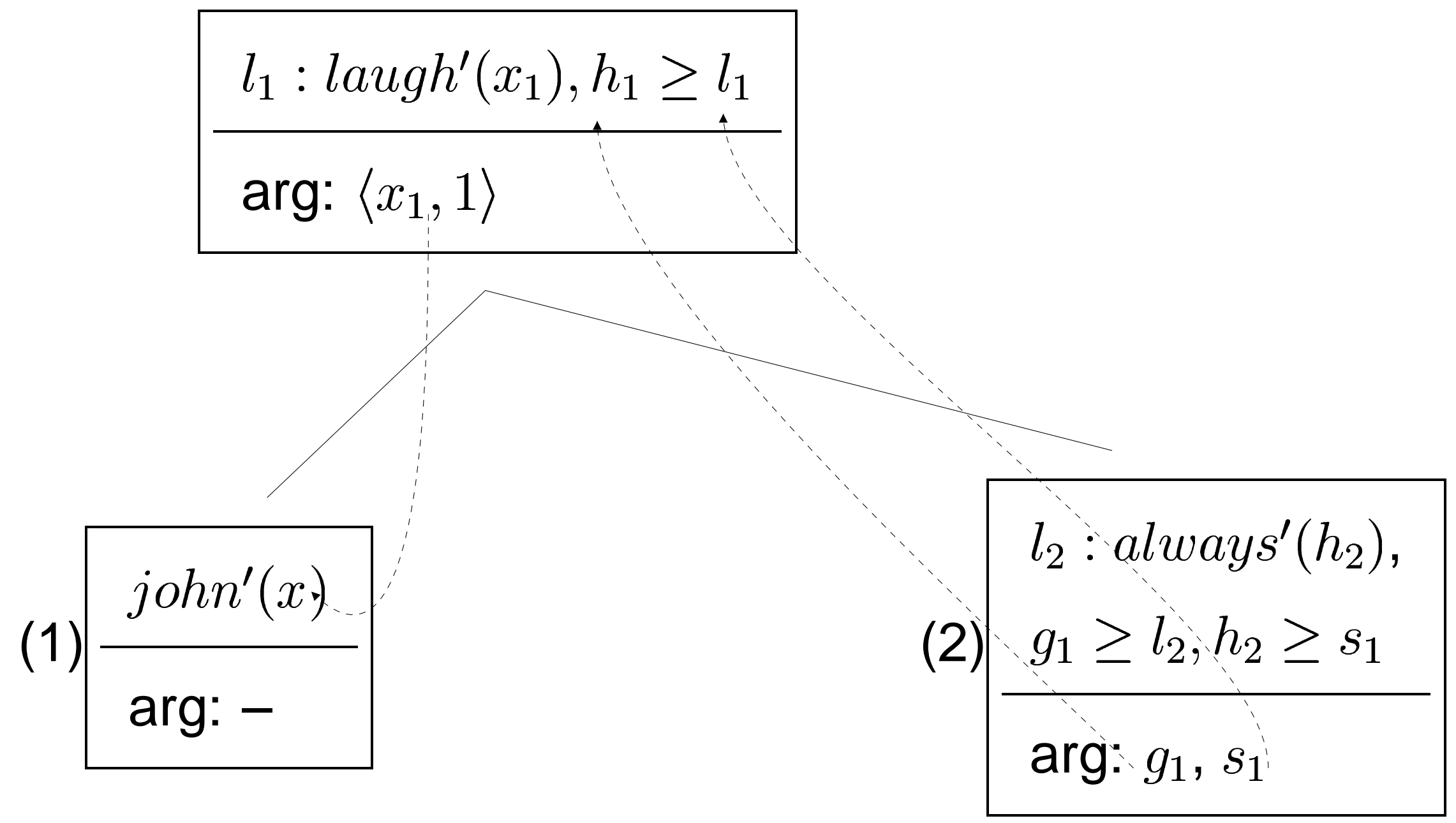




\section{LTAG semantics (3)}

Result: \begin{tabular}{l|}
$l_{1}: \operatorname{laugh}^{\prime}(x)$, john $^{\prime}(x), l_{2}: \operatorname{always}^{\prime}\left(h_{2}\right)$, \\
$h_{1} \geq l_{1}, h_{1} \geq l_{2}, h_{2} \geq l_{1}$ \\
\hline $\arg :-$
\end{tabular} 


\section{LTAG semantics (3)}

Result: \begin{tabular}{l|}
$l_{1}: \operatorname{laugh}^{\prime}(x)$, john $^{\prime}(x), l_{2}: \operatorname{always}^{\prime}\left(h_{2}\right)$, \\
$h_{1} \geq l_{1}, h_{1} \geq l_{2}, h_{2} \geq l_{1}$ \\
\hline arg: -
\end{tabular}

Disambiguation: Bijection from holes to labels such that

(a) subordination on the disambiguated representation is a partial order

(b) no label is subordinated to two labels that are siblings 


\section{LTAG semantics (3)}

Result: \begin{tabular}{l|}
$l_{1}: \operatorname{laugh}^{\prime}(x)$, john $^{\prime}(x), l_{2}: \operatorname{always}^{\prime}\left(h_{2}\right)$, \\
$h_{1} \geq l_{1}, h_{1} \geq l_{2}, h_{2} \geq l_{1}$ \\
\hline $\arg :-$
\end{tabular}

Disambiguation: Bijection from holes to labels such that

(a) subordination on the disambiguated representation is a partial order

(b) no label is subordinated to two labels that are siblings

here: $h_{1} \geq l_{2}>h_{2} \geq l_{1}$, therefore just one disambiguation: $h_{1} \rightarrow l_{2}, h_{2} \rightarrow l_{1} \leadsto \operatorname{john}^{\prime}(x) \wedge \operatorname{always}^{\prime}\left(\operatorname{laugh}^{\prime}(x)\right)$ 


\section{Quantifier scope (1)}

Idea: separating scope and predicate argument information:

(7) every dog barks

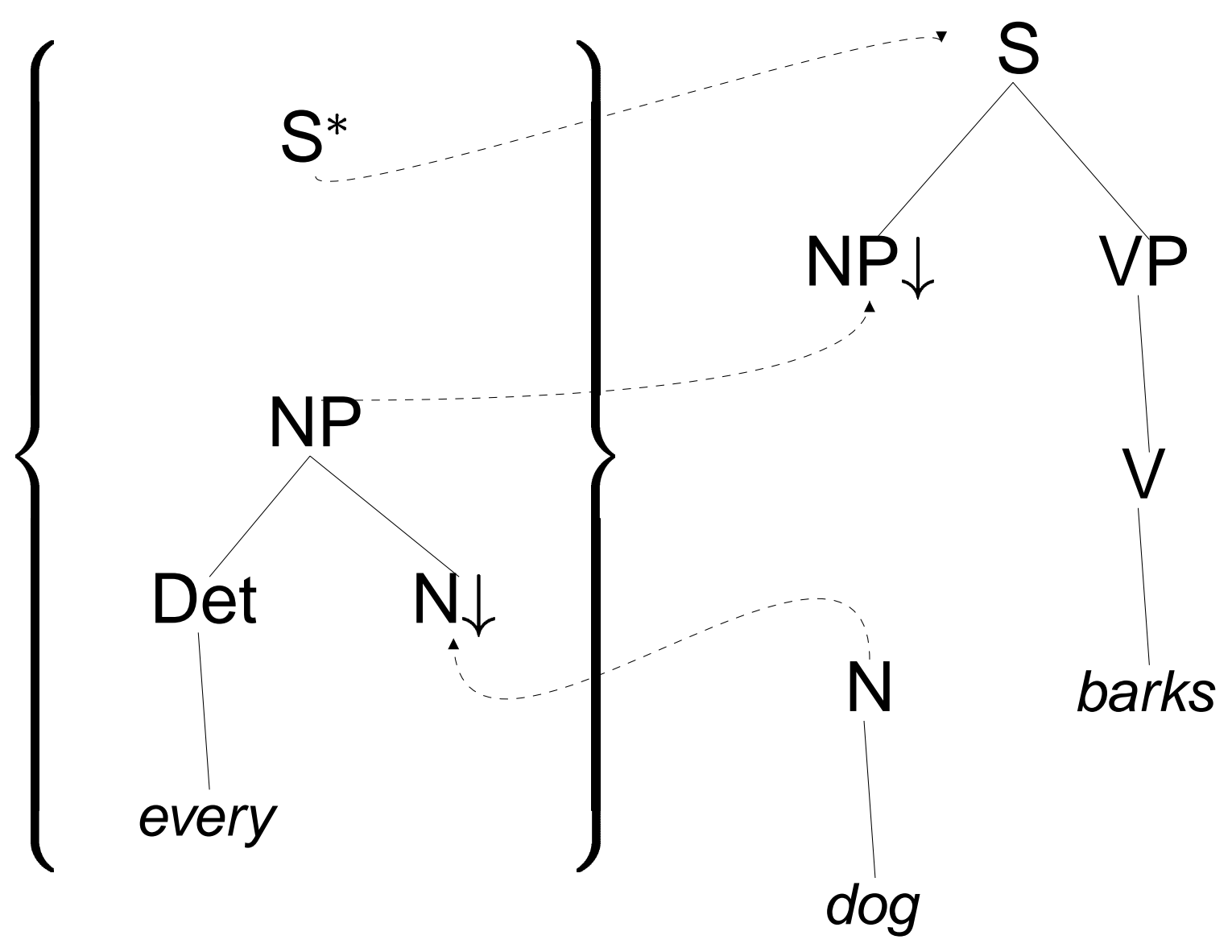




\section{Quantifier scope (2)}

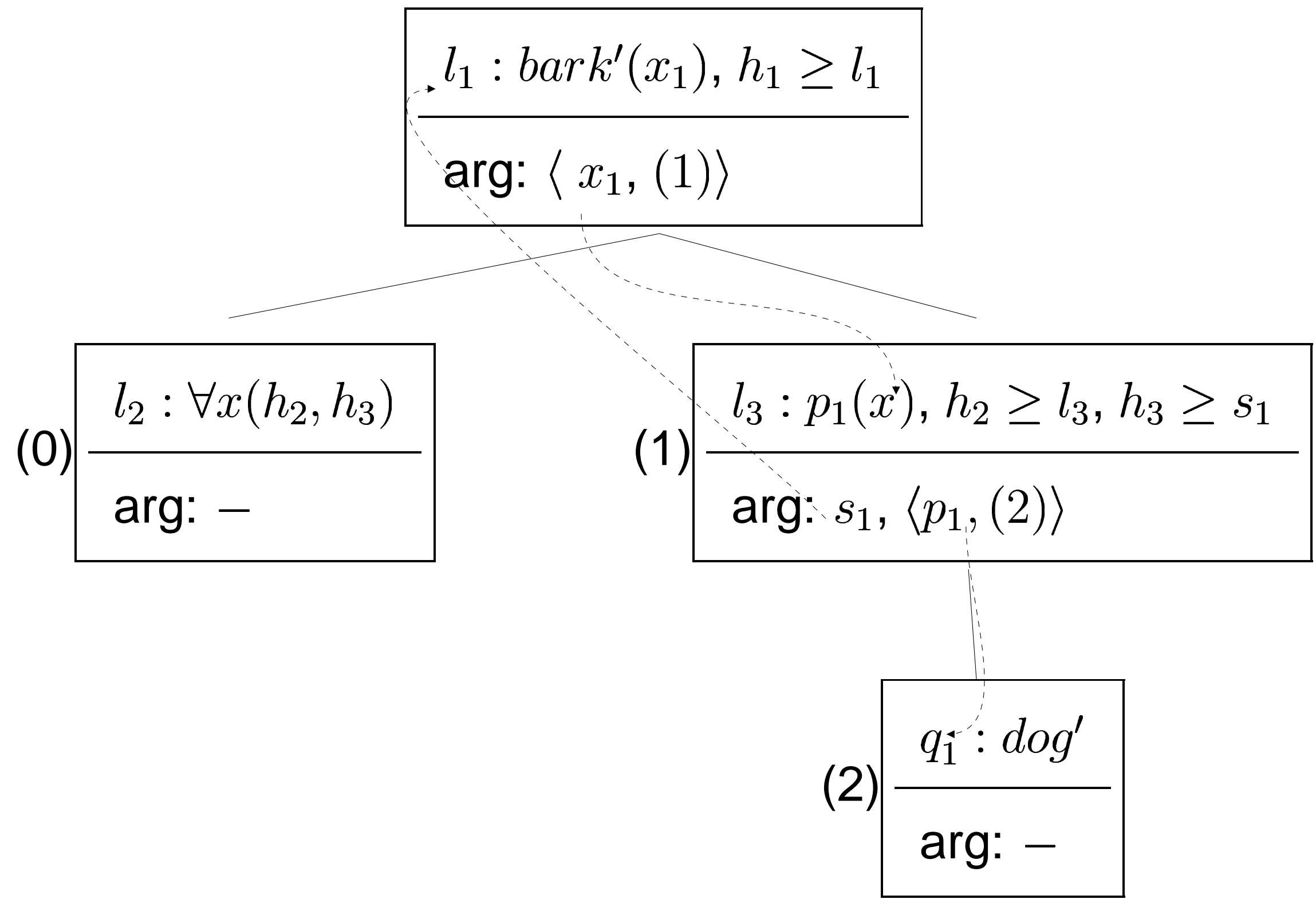




\section{Quantifier scope (3)}

Result: \begin{tabular}{l|}
$l_{1}: \operatorname{bark}^{\prime}(x), l_{2}: \forall x\left(h_{2}, h_{3}\right), l_{3}: \operatorname{dog}^{\prime}(x)$ \\
$h_{1} \geq l_{1}, h_{3} \geq l_{1}, h_{2} \geq l_{3}$ \\
\hline $\arg :-$
\end{tabular}

just one disambiguation:

$$
\begin{aligned}
& h_{1} \rightarrow l_{2}, h_{2} \rightarrow l_{3}, h_{3} \rightarrow l_{1} \\
& \leadsto \forall x\left(\operatorname{dog}^{\prime}(x), \operatorname{bark}^{\prime}(x)\right)
\end{aligned}
$$




\section{Quantifier scope (4)}

Underspecified representations for scope ambiguities:

(8) some student loves every course

$$
\begin{aligned}
& l_{2}: \exists x\left(h_{2}, h_{3}\right), l_{4}: \forall y\left(h_{4}, h_{5}\right), \\
& l_{1}: \operatorname{loves}^{\prime}(x, y), l_{3}: \text { student }^{\prime}(x), l_{5}: \operatorname{course}^{\prime}(y), \\
& h_{2} \geq l_{3}, h_{3} \geq l_{1}, h_{4} \geq l_{5}, h_{5} \geq l_{1}, h_{1} \geq l_{1} \\
& \text { arg: - }
\end{aligned}
$$

two disambiguations:

๖ $h_{1} \rightarrow l_{2}, h_{2} \rightarrow l_{3}, h_{3} \rightarrow l_{4}, h_{4} \rightarrow l_{5}, h_{5} \rightarrow l_{1}$ (wide scope of $\exists$ )

๖ $h_{1} \rightarrow l_{4}, h_{2} \rightarrow l_{3}, h_{3} \rightarrow l_{1}, h_{4} \rightarrow l_{5}, h_{5} \rightarrow l_{2}$ (wide scope of $\forall$ ) 


\section{Flexible composition (1)}

General idea: consider substitutions and adjunctions as attachments that can go in either direction. Flexible composition: attaching a tree $t$ or a set of trees $\left\{t_{1}, \ldots, t_{n}\right\}$ to an elementary tree (or tree set) $u$

- Allows different orders when traversing the derivation tree.

Extends the generative capacity of TAG.

For our purpose only restricted use of flexible composition: standard TAG derivation trees with a bottom-up traversal. (This special case is weakly equivalent to TAG.) 


\section{Flexible composition (2)}

Flexible composition derivation for (2) two politicians spy on someone from every city

1. tree set for from every city is built and it attaches to the tree set for someone

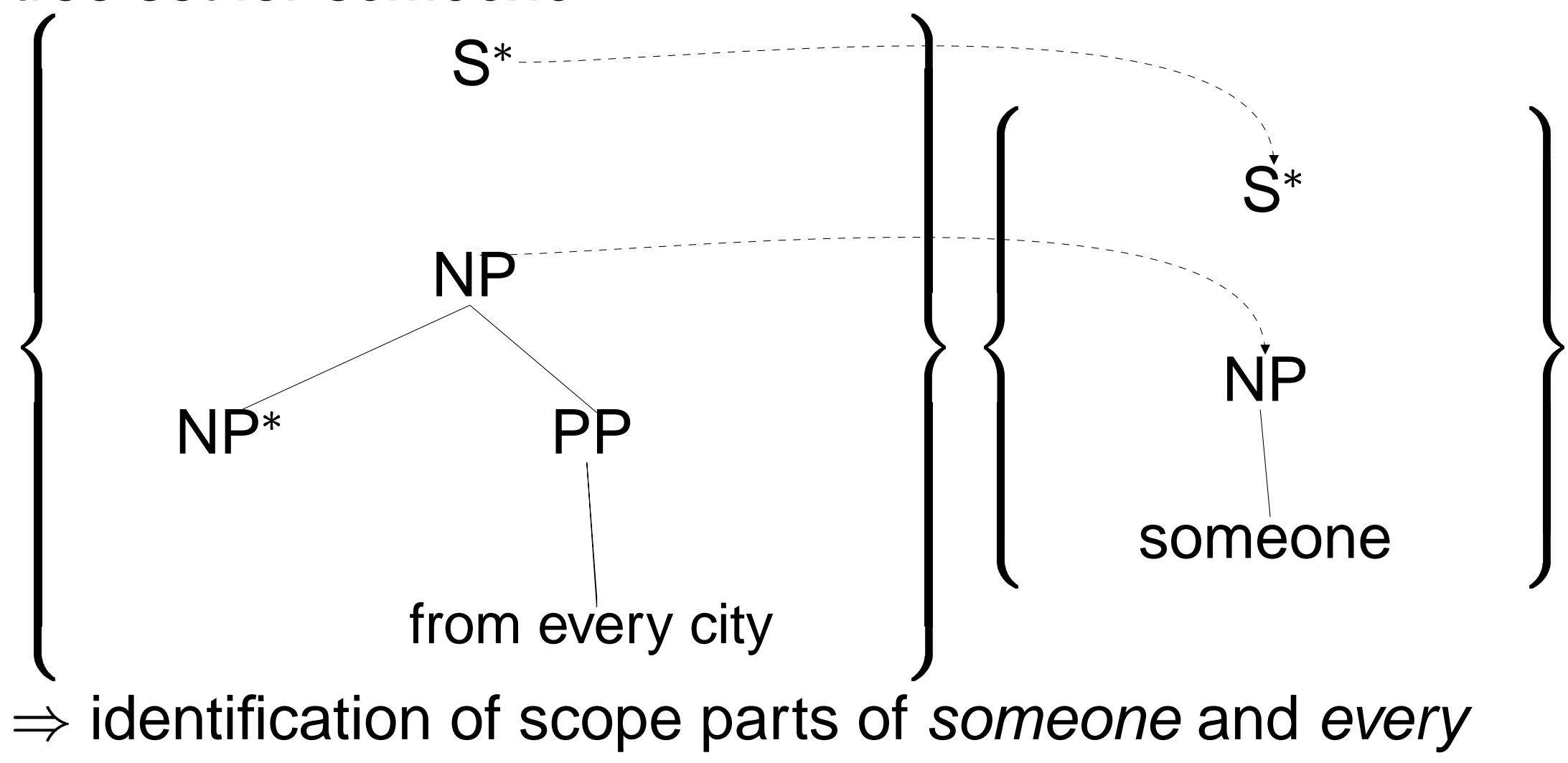




\section{Flexible composition (3)}

2. the tree sets for two politicians and someone from every city attach simultaneously to spy:

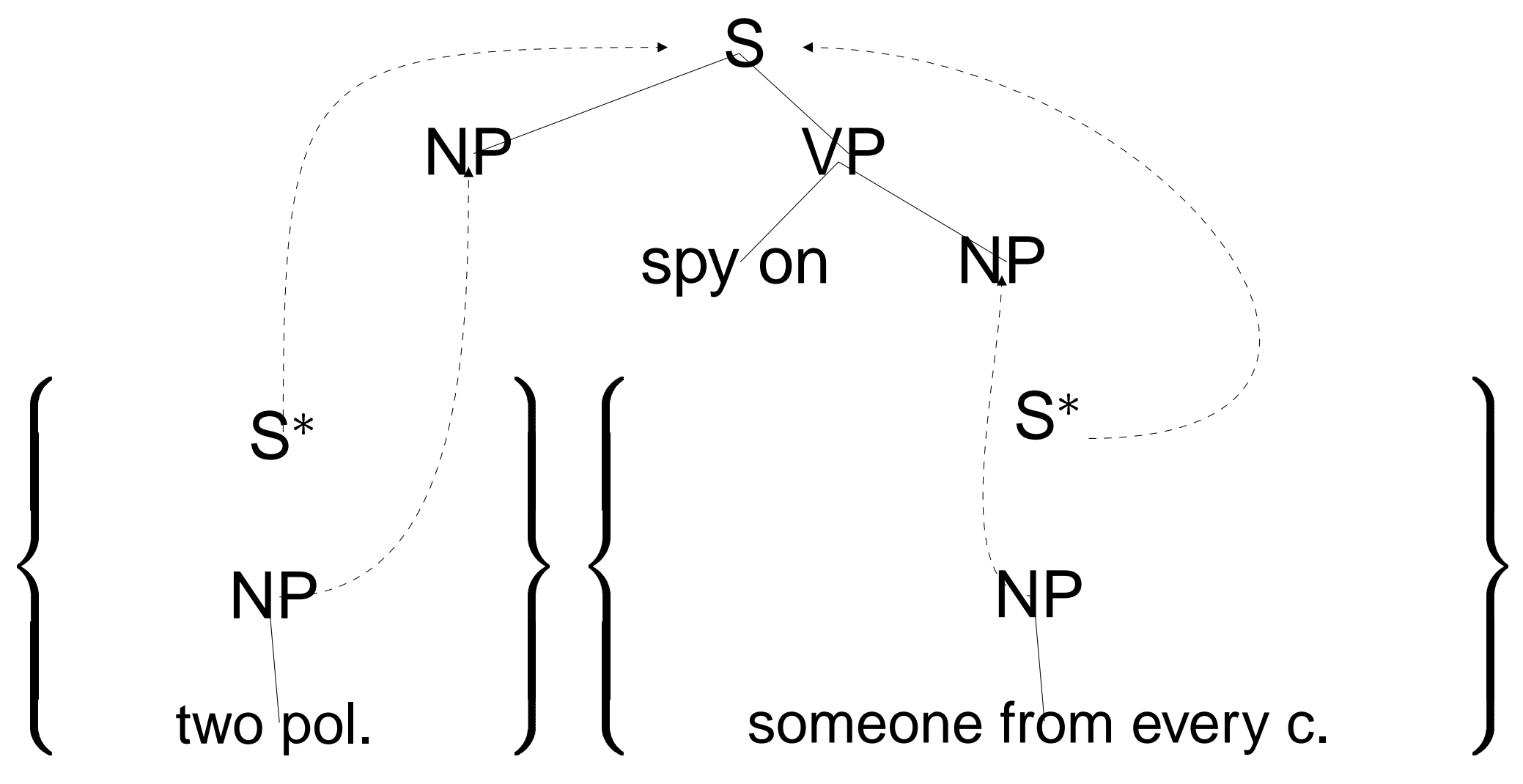

$\Rightarrow$ identification of scope parts of two on the one hand and someone and every on the other hand 


\section{Quantifier set approach (1)}

Observation: whenever an identification of scope parts takes place,

- all scope orders are possible between the quantifier groups involved in that identification, and

\& no other quantifier can intervene between them.

$\Rightarrow$ quantifiers that are identified are 'glued together' such that nothing else can intervene. 


\section{Quantifier set approach (2)}

Formalization with quantifier sets: 


\section{Quantifier set approach (2)}

Formalization with quantifier sets:

( introduce quantifier sets: whenever quantifiers scope trees are identified, a new set is built containing the scope parts of these quantifiers. (Eventually, these scope parts are already sets.) 


\section{Quantifier set approach (2)}

Formalization with quantifier sets:

( introduce quantifier sets: whenever quantifiers scope trees are identified, a new set is built containing the scope parts of these quantifiers. (Eventually, these scope parts are already sets.)

- additional condition on scope order for disambiguated representations:

(c) if one part of a quantifier set $Q_{1}$ is subordinated by one part of another quantifier set $Q_{2}$, then all quantifiers in $Q_{1}$ must be subordinated by all quantifiers in $Q_{2}$. 


\section{Quantifier set approach (3)}

Semantic representation of (2):

$$
\begin{aligned}
& \left\{l_{1}: 2 x\left(h_{1}, h_{2}\right),\left\{l_{3}: \forall y\left(h_{3}, h_{4}\right), l_{6}: \exists z\left(h_{6}, h_{7}\right)\right\}\right\} \\
& l_{2}: \operatorname{politicians}^{\prime}(x), l_{4}: \operatorname{city}^{\prime}(y), l_{5}: \operatorname{from}^{\prime}(z, y), \\
& l_{7}: \operatorname{person}^{\prime}(z), l_{8}: \operatorname{spy}^{\prime}(x, z) \\
& h_{1} \geq l_{2}, h_{2} \geq l_{8}, h_{3} \geq l_{4}, h_{4} \geq l_{5}, h_{5} \geq l_{5}, \\
& h_{6} \geq h_{5}, h_{5} \geq l_{7}, h_{6} \geq l_{7}, h_{7} \geq l_{8}, h_{8} \geq l_{8} \\
& \text { arg:- }
\end{aligned}
$$

Inverse linking reading $\forall 2 \exists=l_{3}>l_{1}>l_{6}$ excluded: For $Q_{1}:=\left\{l_{3}: \forall \ldots, l_{6}: \exists \ldots\right\}$ and $Q_{2}:=l_{1}: 2 \ldots$, the scope order condition (c) would not be satisfied because $l_{3}>l_{1}$ and $l_{6} \ngtr l_{1}$. 


\section{Conclusion}

2 Data: In $\mathrm{Qu}_{1} \ldots\left[\mathrm{Qu}_{2}\left[\mathrm{Qu}_{3}\right]\right]$, the inverse linking reading where $\mathrm{Qu}_{1}$ intervenes between the host $\mathrm{Qu}_{2}$ and the nested $\mathrm{Qu}_{3}$ is impossible: ${ }^{*} \mathrm{Qu}_{3}>\mathrm{Qu}_{1}>\mathrm{Qu}_{2}$.

- Account:

- Using scope parts for quantifiers and flexible composition, quantifier sets are constructed that group argumentally related quantifiers.

- Constraints are imposed on quantifier sets: given two quantifier sets $Q_{1}$ and $Q_{2}$, all the quantifiers in $Q_{1}$ must have the same scopal relation to all the quantifiers in $Q_{2}$.

The flexible composition approach as used here does not increase the weak generative capacity of TAG. 